\title{
Fermi surface of $\mathrm{PtCoO}_{2}$ from quantum oscillations and electronic structure calculations
}

\author{
F. Arnold, ${ }^{1}$ M. Naumann $\odot,{ }^{1,2}$ H. Rosner,${ }^{1}$ N. Kikugawa, ${ }^{3}$ D. Graf, ${ }^{4}$ L. Balicas,${ }^{4}$ T. Terashima, ${ }^{3}$ \\ S. Uji, ${ }^{3,5}$ H. Takatsu ${ }^{\circledR},{ }^{6, *}$ S. Khim $\odot,{ }^{1}$ A. P. Mackenzie, ${ }^{1,7}$ and E. Hassinger ${ }^{1,2, \dagger}$ \\ ${ }^{1}$ Max Planck Institute for Chemical Physics of Solids, 01187 Dresden, Germany \\ ${ }^{2}$ Physik-Department, Technische Universität München, 85748 Garching, Germany \\ ${ }^{3}$ National Institute for Materials Science, Tsukuba, Ibaraki 305-0003, Japan \\ ${ }^{4}$ National High Magnetic Field Laboratory, Florida State University, Tallahassee, Florida 32310, USA \\ ${ }^{5}$ Graduate School of Pure and Applied Sciences, University of Tsukuba, Tsukuba 305-8577, Japan \\ ${ }^{6}$ Department of Physics, Tokyo Metropolitan University, Tokyo 192-0397, Japan \\ ${ }^{7}$ Scottish Universities Physics Alliance, School of Physics and Astronomy, University of St. Andrews, \\ St. Andrews, Fife KY16 9SS, United Kingdom
}

(Received 15 January 2020; revised manuscript received 23 March 2020; accepted 6 April 2020; published 1 May 2020)

\begin{abstract}
The delafossite series of layered oxides includes some of the highest conductivity metals ever discovered. Of these, $\mathrm{PtCoO}_{2}$, with a room-temperature resistivity of $1.8 \mu \Omega \mathrm{cm}$ for in-plane transport, is the most conducting of all. The high conduction takes place in triangular lattice Pt layers, separated by layers of Co-O octahedra, and the electronic structure is determined by the interplay of the two types of layers. We present a detailed study of quantum oscillations in $\mathrm{PtCoO}_{2}$, at temperatures down to $35 \mathrm{mK}$ and magnetic fields up to $30 \mathrm{~T}$. As for $\mathrm{PdCoO}_{2}$ and $\mathrm{PdRhO}_{2}$, the Fermi surface consists of a single cylinder with mainly Pt character and an effective mass close to the free-electron value. Due to Fermi-surface warping, two close-lying high frequencies are observed. Additionally, a pronounced difference frequency appears. By analyzing the detailed angular dependence of the quantum-oscillation frequencies, we establish the warping parameters of the Fermi surface. We compare these results to the predictions of first-principles electronic-structure calculations including spin-orbit coupling on $\mathrm{Pt}$ and $\mathrm{Co}$ and on-site correlation $U$ on $\mathrm{Co}$, and hence demonstrate that electronic correlations in the Co-O layers play an important role in determining characteristic features of the electronic structure of $\mathrm{PtCoO}_{2}$.
\end{abstract}

DOI: 10.1103/PhysRevB.101.195101

\section{INTRODUCTION}

The delafossite series of oxides, with the general formula $\mathrm{ABO}_{2}$, are based on stacks of triangularly coordinated layers with a three-unit periodicity resulting in the point-group symmetry $R \overline{3} m$ for the most common $3 R$ structural isomorph [1]. They host a large number of $\mathrm{AB}$ combinations, with A-site atoms including $\mathrm{Pt}, \mathrm{Pd}, \mathrm{Ag}$, and $\mathrm{Cu}$, and $\mathrm{B}$-site transition metals such as $\mathrm{Cr}, \mathrm{Ge}, \mathrm{Co}, \mathrm{Ni}$, and $\mathrm{Rh}$ in an octahedral coordination with oxygen [2]. The formal valence of the transition metal is $3+$, leading sometimes (e.g., in the case of $\mathrm{Cr}$ ) to a local magnetic moment but in other cases, notably $\mathrm{Co}^{3+}$ in its low-spin $3 d^{6}$ configuration, to nonmagnetic ions. Most delafossites are insulators (often magnetic) and semimetals,

\footnotetext{
*Present address: Department of Energy \& Hydrocarbon Chemistry, Graduate School of Engineering, Kyoto University, Kyoto 6158510, Japan.

${ }^{\dagger}$ Corresponding author: elena.hassinger@cpfs.mpg.de

Published by the American Physical Society under the terms of the Creative Commons Attribution 4.0 International license. Further distribution of this work must maintain attribution to the author(s) and the published article's title, journal citation, and DOI. Open access publication funded by the Max Planck Society.
}

but five $\left(\mathrm{PdCoO}_{2}, \mathrm{PdCrO}_{2}, \mathrm{PdRhO}_{2}, \mathrm{PtCoO}_{2}\right.$, and $\left.\mathrm{AgNiO}_{2}\right)$ are known to be metals [3]. In $\mathrm{AgNiO}_{2}$, the conduction is due to the Ni-O states, and occurs in a complex phase diagram in which conducting phases are interleaved with charge-ordered insulators [4,5]. In contrast, in Pd- and Pt-based materials, the conduction results predominantly from the $4 d-5 s$ or $5 d-6 s$ states of $\mathrm{Pd}$ or $\mathrm{Pt}$, respectively, leading to large bandwidths and almost free-electron-like conduction in these quasi-twodimensional metals. The conductivity is extremely high, with the mean-free path deduced from electrical transport measurements rising from nearly $1000 \AA$ at room temperature to ten microns or more below $10 \mathrm{~K}$ [6-9].

A situation in which nearly free electrons flow in close proximity to layers of transition-metal oxides in which electron correlations are expected to be strong is highly unusual. The delafossite metals have already displayed rich magnetotransport and surface state physics, some of which is a direct result of interlayer coupling [10-16]. An appealing aspect is the simplicity of their bulk Fermi surfaces. In the Pd- and Pt-based cobaltates and rhodates, a single band crosses the Fermi level, and the strongly two-dimensional character of their electronic structure facilitates the mapping of their Fermi surface using angle-resolved photoemission spectroscopy (ARPES) [9,12,17-19]. The very long meanfree paths have enabled detailed de Haas-van Alphen (dHvA) experiments to complement the ARPES data in $\mathrm{PdCoO}_{2}$, 
$\mathrm{PdRhO}_{2}$, and $\mathrm{PdCrO}_{2}[8,19-21]$ but only preliminary dHvA data have been reported for $\mathrm{PtCoO}_{2}$, for fields close to the crystallographic $c$ axis [9]. In this paper, we describe and analyze the results of a comprehensive $\mathrm{dHvA}$ rotation study of $\mathrm{PtCoO}_{2}$. Based on these results, the weak $c$-axis dispersion is modeled, applying similar methods as those previously used for $\mathrm{PdCoO}_{2}$ and $\mathrm{PdRhO}_{2}[8,19]$. Comparing the results to band-structure calculations, including a correlation parameter $U$ on the Co site, we obtain a good agreement for strong correlations $(U=6 \mathrm{eV})$, although the $c$-axis warping is slightly overestimated in the calculations.

\section{EXPERIMENTAL AND COMPUTATIONAL METHODS}

Crystal growth and the characterization of single crystals of $\mathrm{PtCoO}_{2}$ are described in Ref. [22] and references therein. We studied the de Haas- van Alphen oscillations of four $\mathrm{PtCoO}_{2}$ crystals, named $\mathrm{S} 1$ to $\mathrm{S} 4$, by magnetic torque measurements in several laboratories around the globe, with different experimental setups as described below. Samples S2 and S3 were grown by S. Khim and samples S1 and S4 by H. Takatsu.

Sample S1 was studied at National Institute for Material Science in Japan. The magnetic torque was measured using piezoresistive PRC400 microcantilevers in a top-loading type dilution-refrigerator down to $40 \mathrm{mK}$ in a superconducting magnet and magnetic fields of up to $17.5 \mathrm{~T}$.

Samples S2 and S3 were studied at the Max Planck Institute for Chemical Physics of Solids in Germany. The quantum oscillations of S2 and S3, from the same growth batch, were observed at temperatures between $700 \mathrm{mK}$ and $4 \mathrm{~K}$ in magnetic fields up to $15 \mathrm{~T}$. The respective sample sizes were approximately $200 \times 300 \times 50(\mu \mathrm{m})^{3}$ and $150 \times 100 \times$ $20(\mu \mathrm{m})^{3}$. Experiments on these samples were also performed using piezoresistive PRC400 microcantilevers, installed on a MX400 Oxford Instruments dilution refrigerator with a $15 / 17 \mathrm{~T}$ superconducting magnet and a $270^{\circ}$ Swedish rotator with an angular accuracy of $\Delta \theta= \pm 0.2^{\circ}$. The magnetometer utilizes a two-stage dc-SQUID (superconducting quantuminterference device) as highly sensitive readout, offering a torque resolution of $\Delta \tau=2 \times 10^{-13} \mathrm{Nm}$ at lowest temperatures $[23,24]$. Data were taken at constant temperatures whilst the magnetic field was swept from 15 to $7.5 \mathrm{~T}$ at a rate of $30 \mathrm{mT} / \mathrm{min}$. Sample S2 was measured in two orientations so the field was rotated in the crystallographic $\mathrm{Z} \Gamma \mathrm{K}$ and $\mathrm{Z} \Gamma \mathrm{L}$ planes.

Sample S4 was studied at the National High Magnetic Field Laboratory in the USA. Torque magnetometry was measured by using the capacitive method with a 0.025 -mm-thick $\mathrm{CuBe}$ lever in a ${ }^{3} \mathrm{He}$ cryostat and a resistive magnet producing fields up to $35 \mathrm{~T}$ and a top-loading type dilution refrigerator in a superconducting magnet generating fields up to $16 \mathrm{~T}$. The magnetic field direction in all experiments is given with respect to the crystallographic $c$ axis.

Relativistic density-functional (DFT) electronic-structure calculations were performed using the full-potential FPLO code $[25,26]$, version fplo18.00-52. For the exchangecorrelation potential, within the generalized gradient approximation (GGA), the parametrization of Perdew-BurkeErnzerhof [27] was chosen. The spin-orbit coupling was treated nonperturbatively, solving the four component Kohn-
Sham-Dirac equation [28]. To obtain precise band-structure information, the calculations were carried out on a wellconverged mesh of $125000 k$ points $(50 \times 50 \times 50$ mesh, 11076 points in the irreducible wedge of the Brillouin zone). The Fermi surface was calculated on a self-adjusting $k$ mesh to ensure maximum accuracy. The Coulomb repulsion in the Co-3d shell was simulated in a mean-field way, applying the $\mathrm{GGA}+U$ approximation in the atomic-limit-flavor. For all calculations, the experimental crystal structure [1] was used.

\section{RESULTS AND DISCUSSION}

\section{A. Quantum oscillations in $\mathrm{PtCoO}_{2}$}

Typical quantum oscillations in the magnetic torque at different angles for samples S2 and S4 are shown in Fig. 1. Data from all high-quality samples reported in this paper are mutually consistent. The oscillations consist mainly of three frequencies. Two close-lying high frequencies, $F_{\alpha}$ and $F_{\beta}$, lead to fast oscillations of the signal with varying amplitude due to beating. As expected, the beating frequency is half the difference between the two high frequencies $F_{\alpha}$ and $F_{\beta}$. Additionally, we also see strong quantum oscillations with a "slow" frequency $F_{\gamma}$, where $F_{\gamma}=F_{\beta}-F_{\alpha}$ at all angles as shown in Fig. 4. This situation is reminiscent of, but even more pronounced than in, $\mathrm{PdCoO}_{2}$ [8]. There, the slow oscillations have been ascribed to magnetic interaction, meaning that the oscillations (of magnetization, chemical potential, or density of states) induced by the field couple back to the Landau level structure as a second-order effect, creating combination frequencies [29]. Torque interaction, which can have a similar experimental signature, was ruled out since the slow oscillations also appear in the resistance $[8,29]$. The same is true for $\mathrm{PtCoO}_{2}$ [13]. However, $\mathrm{PdRhO}_{2}$ with a very similar Fermi surface does not show the slow oscillations [19]. At some angles, additional variations of the oscillation amplitudeslower than the dominant beating caused by $F_{\alpha}$ and $F_{\beta}$-hint at smaller splittings of the main frequencies, also observed in the fast Fourier transforms (FFTs). For example, the FFT of the torque data at $4^{\circ}$ in Fig. 1(c) shows two double peaks. The reason for this is not understood but, nevertheless, two main peaks could always be identified. Overall, the fast quantumoscillation frequencies follow a $1 / \cos \theta$ angular dependence consistent with a cylindrical quasi-two-dimensional Fermi surface as also observed in $\mathrm{PdCoO}_{2}$ and $\mathrm{PdRhO}_{2}[8,19]$.

\section{B. Properties for $B \| c$}

For $B \| c$, the main frequencies are $F_{\alpha}=29.92 \mathrm{kT}$ and $F_{\beta}=30.49 \mathrm{kT}$ with a difference frequency of $F_{\gamma}=0.57 \mathrm{kT}$.

The measured mean quantum-oscillation frequency of $\bar{F}_{0}=30.20 \pm 0.01 \mathrm{kT}$ results in a Fermi-surface cross section of $S_{\text {ext }}=2.883 \AA^{-2}$. The cross section multiplied by the height of the Brillouin zone gives the Fermi-surface volume, which is, according to Luttinger's theorem, directly related to the carrier density. Given the lattice parameters of $a=2.82 \AA$ and $c=17.808 \AA$ [9], the experimental Fermi-surface cross section is only $0.6 \%$ larger than the cross section from a half-filled metal and hence corresponds to a Luttinger count of 1.006. A half-filled metal is expected when each Pt atom contributes one electron to the conduction band. Using a 

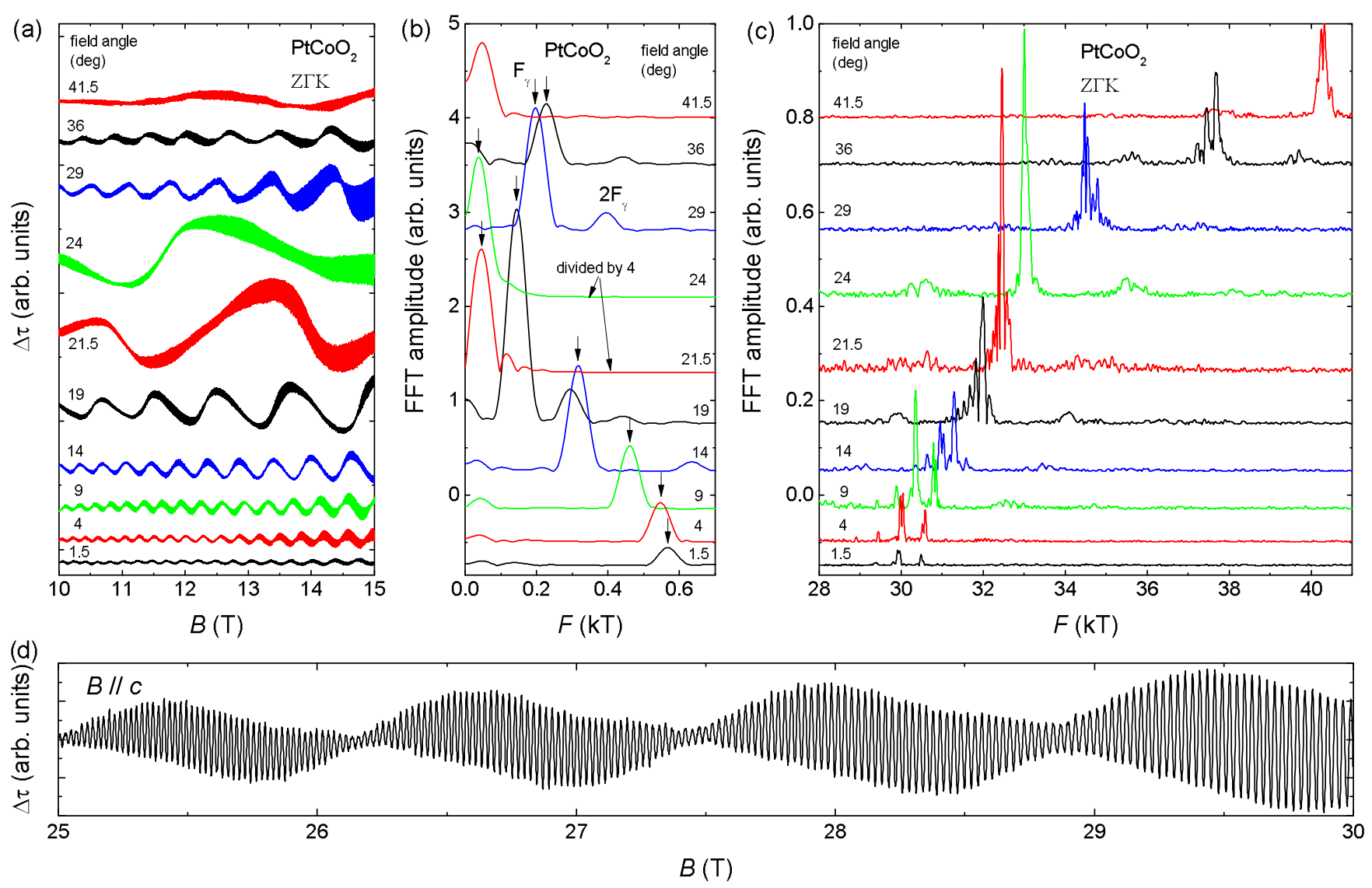

FIG. 1. (a) Exemplary de Haas-van Alphen oscillations in the magnetic torque of the $\mathrm{PtCoO}_{2}$ sample $\mathrm{S} 2$ measured at about $700 \mathrm{mK}$ and in magnetic field applied at indicated angles away from $B \| c$ within the Z $\Gamma \mathrm{K}$ plane. The dHvA signals were obtained by subtracting the paramagnetic background via a second-order polynomial from the raw magnetic torque signal. (b), (c) Fast Fourier transforms (FFT) were taken between 10 and $15 \mathrm{~T}$ after multiplying the data by a Hanning window. The strong difference frequency $F_{\gamma}$ is visible both in the oscillatory signal (a) as well as the FFT (b). d) High-field torque oscillations of sample S4 at $370 \mathrm{mK}$ and the magnetic field $B$ around $1^{\circ}$ from the $c$ axis.

slightly larger lattice parameter from Ref. [1] of $a=2.83 \AA$ (the value used for the DFT calculations below), the Fermisurface cross section is $1.4 \%$ larger than the half-filled value. We note, however, that these lattice parameters are based on room-temperature $\mathrm{x}$-ray diffraction, and that thermal contraction likely means that our true Luttinger count is close to 1.

By measuring the temperature dependence of the quantumoscillation amplitude from sample S2, we extract the cyclotron masses for the three dominant frequencies close to $B \| c$, as shown in Fig. 2. Fitting the data by the LifshitzKosevich temperature reduction term yields the cyclotron masses $m_{\alpha}=(1.0 \pm 0.1) m_{0}, m_{\beta}=(1.1 \pm 0.1) m_{0}$ and $m_{\gamma}=$ $(2.0 \pm 0.2) m_{0}$ where $m_{0}$ is the free-electron mass. These values are slightly lower than the effective masses from ARPES [9], where a mean effective mass of $m=1.18 m_{0}$ was found. ARPES results show that the effective mass is $k$ dependent within the plane, and dominated by the variation of $k_{\mathrm{F}}$ in the hexagonally shaped Fermi-surface cross section. A $k$ dependence also along the $c$ direction (as calculated below) might explain the difference between masses from ARPES and those from quantum oscillations which give mean effective masses around orbits at different $k_{\mathrm{z}}$ positions in the Brillouin zone.

At higher fields ( 25 to $30 \mathrm{~T}$ ), the data from sample $\mathrm{S} 4$ give effective masses $m_{\alpha}=(1.1 \pm 0.1) m_{0}, m_{\beta}=(1.2 \pm 0.1) m_{0}$, and $m_{\gamma}=(2.0 \pm 0.2) m_{0}$ that are slightly higher than those determined for sample $\mathrm{S} 2$ but in agreement with the low-field values within the respective uncertainties.

Combining the measured quantum-oscillation frequencies with the cyclotron masses, we obtain the averaged Fermi velocities $v_{\mathrm{F}}=\hbar k_{\mathrm{F}} / m^{\star}$ as $v_{\mathrm{F} \alpha}=(1.1 \pm 0.1) \times 10^{6} \mathrm{~m} / \mathrm{s}$ and $v_{\mathrm{F} \beta}=(1.0 \pm 0.1) \times 10^{6} \mathrm{~m} / \mathrm{s}$ being as high as in simple metals [30] and in reasonable agreement with the directionindependent Fermi velocity from ARPES of $(0.89 \pm 0.09) \times$ $10^{6} \mathrm{~m} / \mathrm{s}$.

A rough estimate of the Dingle temperature $T_{\mathrm{D}}$ was obtained for sample $\mathrm{S} 2$ near $B \| c$ by cutting the total inverse field range (from $1 / 15 \mathrm{~T}$ to $1 / 7.5 \mathrm{~T}$ ) in small equal windows and by taking the FFT of the data in each small window. Since the windows still range over several beating periods of the two high frequencies, the FFT can resolve the two peaks and we extract the field dependence of the FFT amplitude (height) of each peak. A standard Dingle plot then reveals $T_{\mathrm{D}} \approx 3.5 \pm 1.5 \mathrm{~K}$ for both main frequencies [29]. The careful error bar here includes the uncertainty of the effective mass as well as variations of $T_{\mathrm{D}}$ when the field-window size is changed. In Ref. [19], the Dingle temperature was determined via the width of the FFT peak $\Delta F$. This method gives the same values for the Dingle temperature in $\mathrm{PtCoO}_{2}$ if a corrected formula is applied: $\Delta F=T_{\mathrm{D}} \pi^{2} m^{\star} k_{\mathrm{B}} / \mu_{\mathrm{B}}$. Using the relations $T_{\mathrm{D}}=\frac{\hbar}{2 \pi k_{\mathrm{B}} \tau}$ and $v_{\mathrm{F}}=\hbar k_{\mathrm{F}}=l_{0} / \tau$, we obtain a mean-free path 

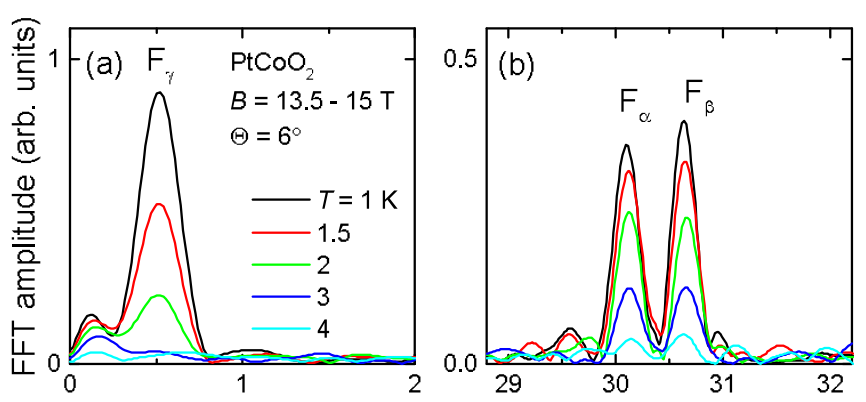

FFT frequency $(\mathrm{kT})$
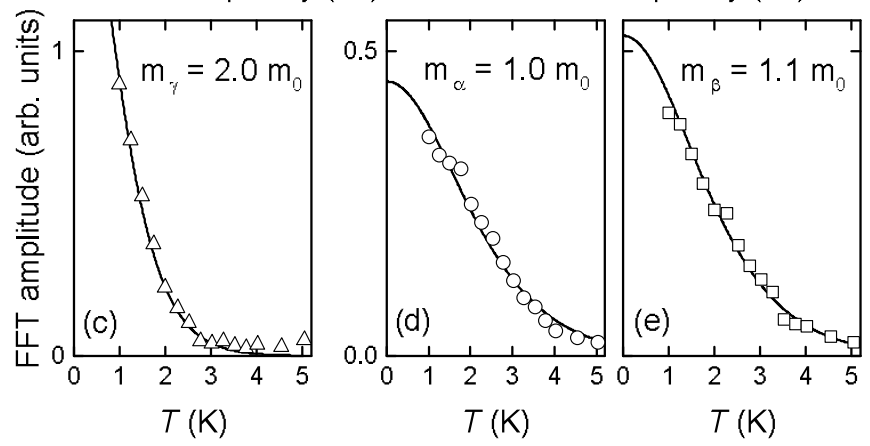

FIG. 2. (a), (b) FFT amplitude for fields in the range $13.5 \mathrm{~T}$ to $15 \mathrm{~T}$ at a magnetic field angle of $6^{\circ}$ off the $c$ axis in the Z $\Gamma \mathrm{L}$ plane and temperatures as indicated. (c)-(e) Temperature dependence of the oscillation peak amplitudes (markers). Lifshitz-Kosevich fits (lines) give the effective masses as indicated within an uncertainty of $10 \%$, where $\mathrm{m}_{0}$ is the free-electron mass.

$l_{0}$ of roughly $350 \pm 150 \mathrm{~nm}$. It is worth noting that the meanfree path obtained here is at least an order of magnitude smaller than the one extracted from the residual resistivity values of samples from the same batch [9] of around $5 \mu \mathrm{m}$. The larger mean-free path in the resistivity might be related to the hexagonal shape of the Fermi surface with large flat portions. There, small-angle scattering does not change the Fermi velocity and hence has little effect on the resistivity. However, all scattering events lead to Landau-level broadening and the subsequent suppression of the quantum-oscillation amplitude. The amplitude of $F_{\gamma}$ also follows a Lifshitz-Kosevich field dependence. The corresponding Dingle temperature is of the order $0.5 \mathrm{~K}$.

\section{Angular dependence of quantum oscillations}

The main quantum-oscillation frequencies $F_{\alpha}$ and $F_{\beta}$ follow roughly a $F / \cos \theta$ angle dependence as expected for a cylindrical Fermi surface, with $F$ being the frequency for $B \| c$. In Fig. 3, the measured frequencies are multiplied with $\cos \theta$ and hence vary with the angle around the constant $\bar{F}_{0}$ obtained for $B \| c$. Note that the jumps/scatter in the angular dependence are due to small uncertainties of the angle. A typical error of $0.2^{\circ}$ at an angle of $40^{\circ}$ results in variations of the factor $\cos \theta$ of around $0.3 \%$ and can explain the jumps of around $100 \mathrm{~T}$ [19]. In addition, a slight backlash of our rotator is evidenced by a departure from the $1 / \cos \theta$ behavior at large angles. In Fig. 3(b), the mean value $\bar{F}_{0}=1 / 2\left(F_{\alpha}+F_{\beta}\right)$ is slightly lower in sample $\mathrm{S} 3$ with respect to sample $\mathrm{S} 2$, perhaps indicating a tiny variation of less than $0.1 \%$ in electron count
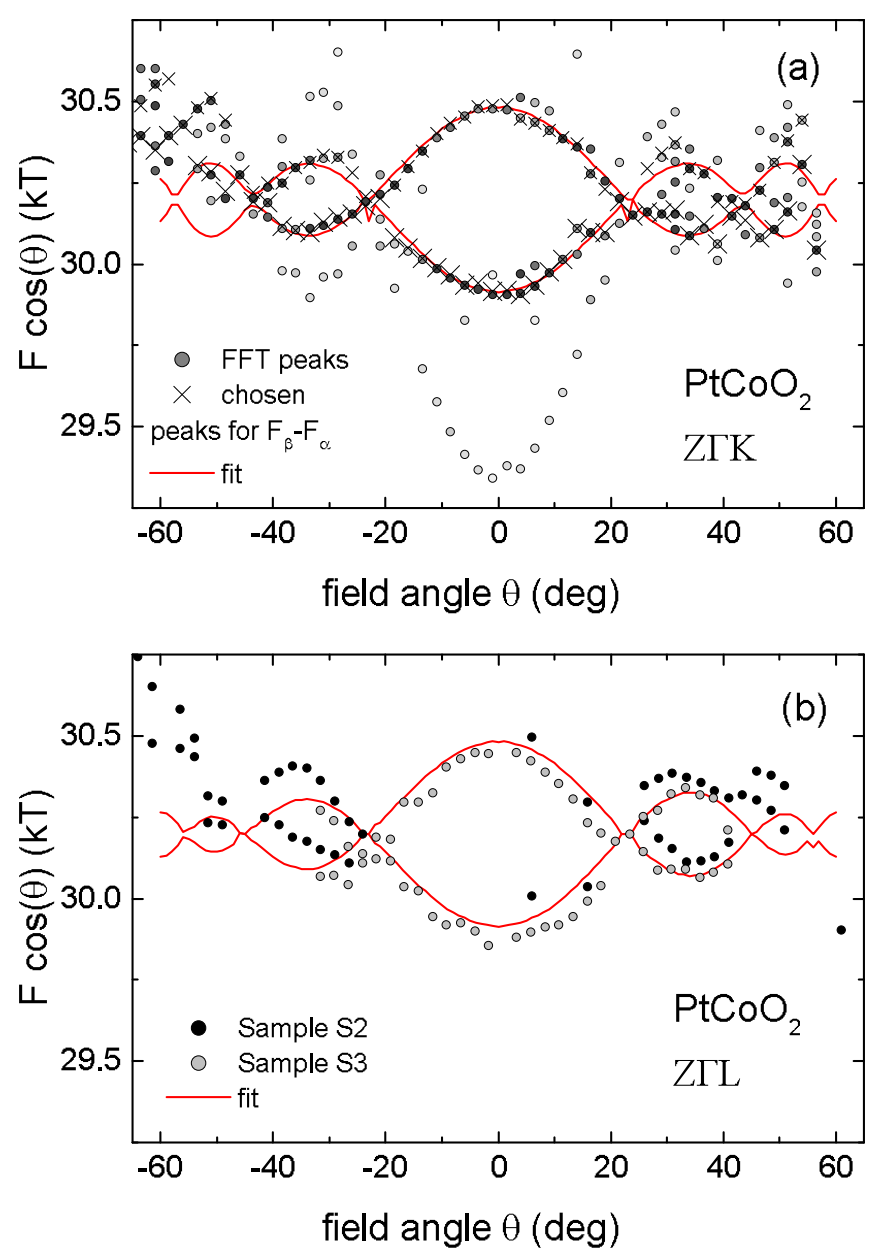

FIG. 3. Angular dependence of the main quantum-oscillation frequencies multiplied by $\cos \theta$ for the two tilting planes $\mathrm{Z} \Gamma \mathrm{K}$ (a) and $\mathrm{Z} \Gamma \mathrm{L}$ (b). The experimental values are to be compared with the angular dependence of a Fermi surface with the warping parameters given in Fig. 5 (red lines). In (a), the peaks in the FFT spectrum taken on data between 10 and $15 \mathrm{~T}$ of sample S2 (dots) were found by an algorithm searching for local maxima. The relative peak height with respect to the maximum peak height of $\alpha$ or $\beta$ peaks is also given by a grey shade of the dots with black being the maximum height at that angle. Peak splittings and side peaks with $F=F_{\alpha}-F_{\gamma}$ or $F=F_{\beta}+F_{\gamma}$ also appear as visible in Fig. 1(c), leading to additional branches. Crosses indicate the peaks for the $\alpha$ and $\beta$ orbits used for the calculation of the difference frequencies $F_{\beta}-F_{\alpha}$ in Fig. 4 when split peaks appeared. Some of these peaks slipped through the algorithm when the peak prominence was too small. In (b), only the peaks corresponding to the crosses in (a) are shown without amplitude shading. Black and grey dots give the angular dependence extracted from samples $S 2$ and $S 3$, respectively.

between both samples. Deviations of the quantum-oscillation frequency from the mean frequency $\bar{F}_{0}$ indicate a departure of the Fermi surface from an ideal cylindrical shape due to a warping that leads to a splitting of the extremal cyclotron orbits into neck and belly orbits. At certain angles of the magnetic field, so-called Yamaji angles, the splitting reduces to zero when all cyclotron orbits inclose the same cross section. In principle, some warping parameters (such as a double $c$-axis warping $-k_{0,2}$ below and in Fig. 5-or strong 

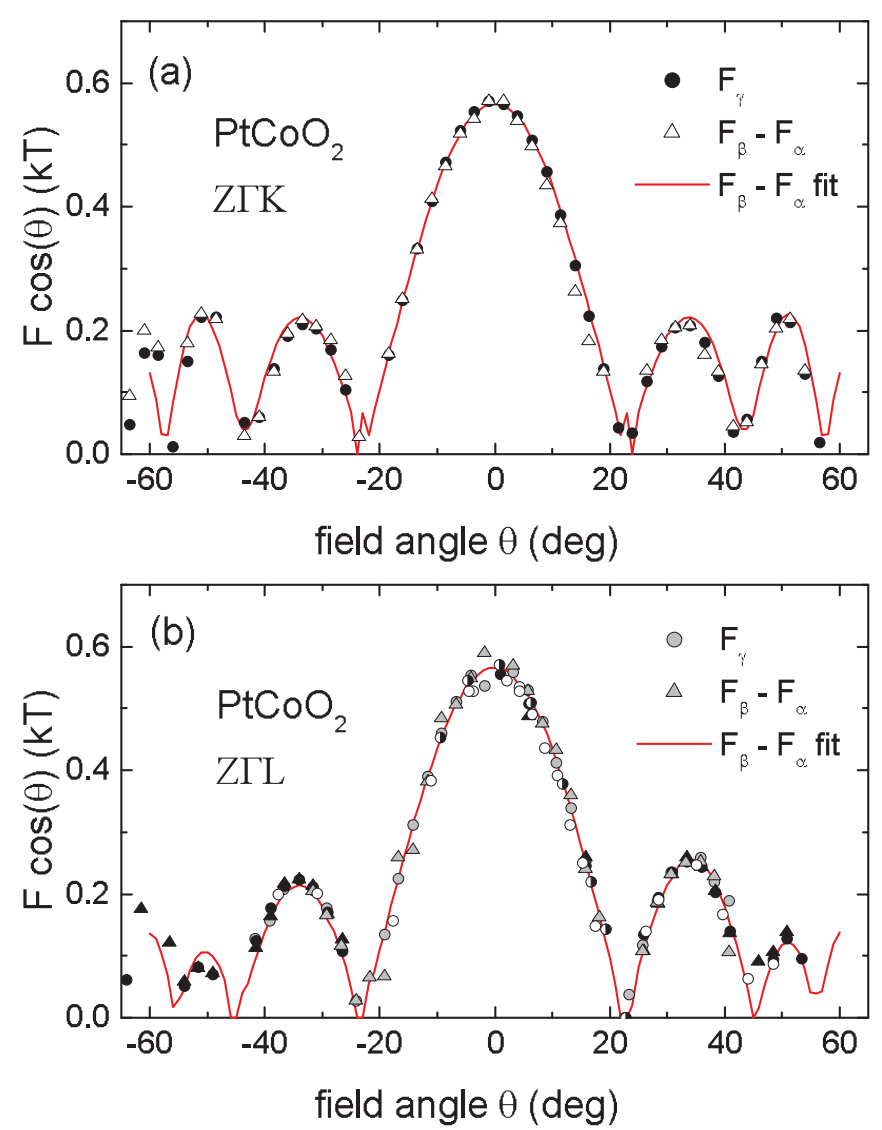

FIG. 4. Angular dependence of the quantum-oscillation frequency $F_{\gamma}$ (dots) and the difference $F_{\beta}-F_{\alpha}$ (triangles) in $\mathrm{PtCoO}_{2}$ for magnetic fields within the ZГK (a) and ZГL plane (b) compared with the values from the approximated Fermi surface of Fig. 5 (red line). In a) experimental data are from sample $S 2$, in b) black symbols are from sample S2, grey symbols from sample S3, white symbols from sample S4 and half black symbols from sample S1.

bumps as in Figs. 6 and 8 for small $U$ ) can cause a departure of the mean frequency from the $1 / \cos \theta$ dependence. However, because of the scatter in the raw data, we cannot confirm or rule out the presence of small contributions of this type.

The angular dependence of $F_{\gamma}$ is given in Fig. 4. It displays exactly the same behavior as the difference between the two main frequencies $F_{\beta}-F_{\alpha}$, supporting that $F_{\gamma}$ stems from magnetic interaction of $F_{\beta}$ and $F_{\alpha}$. Compared to the raw data, the scattering of the $\cos \theta$ factor due to angle uncertainties has a much smaller influence here (triangular symbols in Fig. 4). In this plot, the information on warping causing a departure of the mean frequency with respect to the $1 / \cos \theta$ dependence mentioned above is lost.

The difference frequency $F_{\gamma}$ is reminiscent of the "slow" oscillations observed in quasi-two-dimensional organic conductors [31], although there are some differences. In the organic conductors, the slow oscillations appear only in the interlayer conductivity and are absent in the magnetic torque. They are in good agreement with a theory where fast oscillations in the density of states are mixing with fast oscillations of the chemical potential or Dingle temperature. This leads to interference effects and hence combination frequencies [32] in the conductivity. A similar mixing can also lead to

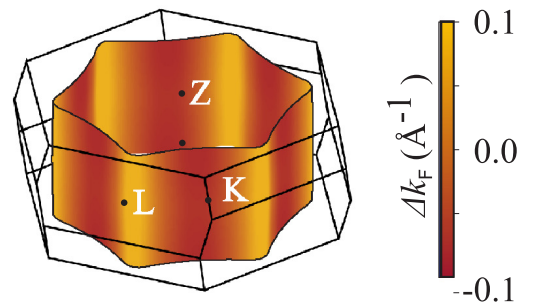

Cylindrical Harmonic Expansion Parameters $k_{00}=0.9581(2) \AA^{-1}$

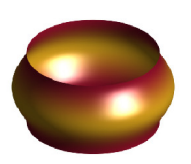

$k_{0,1}$

$0.0045(1) \AA^{-1}$ dHvA

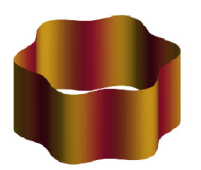

$k_{6,0}$

$0.073(1) \AA^{-1}$

ARPES[9]

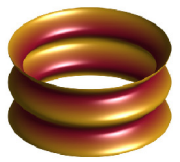

$k_{0,2}$

$0.0000(2) \AA^{-1}$ dHvA

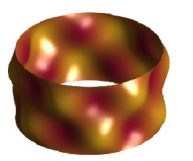

$k_{6,1}$

$0.0013(1) \AA^{-1}$

dHvA

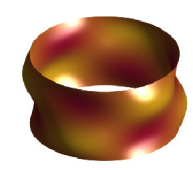

$k_{3,1}$

$0.0004(1) \AA^{-1}$ dHvA
FIG. 5. Reconstructed Fermi-surface topography and cylindrical harmonic expansion parameters of $\mathrm{PtCoO}_{2}$.

slow oscillations in the magnetization as a form of magnetic interaction $[29,33]$. The reason why slow oscillations in the magnetization appear only in the delafossites (and not in the organics) might be the very high purity of these systems but we cannot rule out other origins for their observation. The

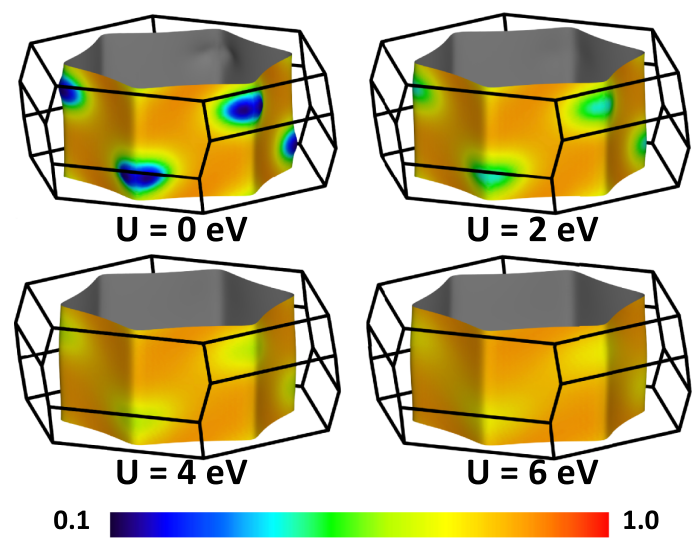

FIG. 6. Impact of the Coulomb parameter $U$, used to describe the strong correlations in the Co $3 d$ orbitals, on the DFT-calculated Fermi surface. The related Fermi velocities are shown by color coding (in $10^{-6} \mathrm{~m} / \mathrm{s}$ ). Without $U$, the Fermi surface exhibits "bumps" incompatible with the experimental observations. With increasing $U$, the "bumps" disappear together with the related strong inhomogeneity of the Fermi velocities. 
absence of the slow frequency in $\mathrm{PdRhO}_{2}$ remains a mystery, and presents a challenge to possible interpretations.

The degree of deviation from two-dimensionality can be estimated via the $c$-axis hopping parameter $t_{\perp}$. It is given by $2 t_{\perp}=\varepsilon_{\mathrm{F}_{\beta}}-\varepsilon_{\mathrm{F}_{\alpha}}$ using the Fermi energy $\varepsilon_{\mathrm{F}}=\hbar^{2} k_{\mathrm{F}}^{2} / 2 \bar{m}^{\star}$ with Planck's constant $\hbar$ and the mean effective mass $\bar{m}^{\star}=1.05 m_{0}$ in units of the free-electron mass $m_{0}$. The Fermi energies $\varepsilon_{\mathrm{F}}$ are then directly related to the quantum-oscillation frequencies by the Onsager relation $F=\frac{\hbar}{2 \pi e} A_{\text {ext }}$ with the extremal Fermi-surface area in reciprocal space $A_{\mathrm{ext}}=\pi k_{\mathrm{F}}^{2}$ and the electron charge $e$. Hence we obtain $t_{\perp}=\frac{\hbar e}{\bar{m}^{*}} \Delta F=31 \mathrm{meV}$. We can expect a two-dimensional behavior when the Landaulevel splitting $\hbar \omega_{c}$ is larger than the dispersion along $c$. We calculate $\hbar \omega_{c}=\hbar e B / \bar{m}^{\star}$ at $15 \mathrm{~T}$ and obtain $\hbar \omega_{c}=1.7 \mathrm{meV}$, a factor of 18 smaller than $t_{\perp}$. This implies that a field of $270 \mathrm{~T}$ would be needed to reach perfect two-dimensionality in this compound.

\section{Fermi-surface warping parameters}

We extract the Fermi-surface warping parameters from the deviation of the two high frequencies from the $1 / \cos \theta$ angular dependence. To model the experimental angular dependence a cylindrical harmonic expansion is used $[8,19,34,35]$,

$$
k_{\mathrm{F}}=\sum_{\mu, v \geqslant 0} k_{\mu, \nu} \cos (v \kappa) \cos (\mu \phi),
$$

where $\kappa=c^{*} k_{z}$ is the reduced $z$ coordinate and $\phi$ the azimuthal angle [19]. Note that the interlayer spacing $c^{*}$ is a third of the $c$ axis lattice parameter. The hexagonal lattice symmetry and $R \overline{3} m\left(D_{3 d}^{5}\right)$ space group limit the allowed $k_{\mu, \nu}$ to $(\mu, v) \in\{(0,0) ;(0,1) ;(0,2) ;(0,3) ;(3,1) ;(6,0) ;(12,0)\}$ and higher order terms. The $k_{\mu \nu}$ given in Fig. 5 (without $k_{6,0}$ and $k_{12,0}$ ) can reproduce the experimental angular dependence in both field-tilting planes (red lines in Figs. 3 and 4). The in-plane parameters $k_{6,0}$ and $k_{12,0}$ do not affect the splitting of the quantum-oscillation frequencies and are therefore invisible to this technique. They were obtained from the Fermisurface shape determined by ARPES [9].

Let us compare the Fermi-surface parameters of $\mathrm{PtCoO}_{2}$ with $\mathrm{PdCoO}_{2}$ [8]. Both have very similar room-temperature lattice parameters (the in-plane lattice parameter $a$ is $2.82 \AA$ in $\mathrm{PtCoO}_{2}$ compared to $2.83 \AA$ in $\mathrm{PdCoO}_{2}$ and the interplane lattice parameter $c$ is $0.4 \%$ larger in $\mathrm{PtCoO}_{2}$ with $17.808 \AA$ compared to $17.73 \AA$ A. On the one hand, $\mathrm{PtCoO}_{2}$ has a much smaller splitting of the main frequency, leading to a $k_{0,1}$ smaller by a factor of 2.4 than in $\mathrm{PdCoO}_{2}$. This points to little direct overlap of the Pt $5 d$ orbitals along the $c$ direction, the value being similar to $\mathrm{PdRhO}_{2}$ [19]. On the other hand, $k_{3,1}$ is of the same order for both compounds (and an order of magnitude smaller than in $\mathrm{PdRhO}_{2}$ ). In $\mathrm{PdCoO}_{2}$, the DFT calculations without correlations on the Co site showed a too-high hybridization between the conduction electrons and the Co electrons and consequently a high $k_{3,1}$ (similar to the bumps in Fig. 6 for $U<4 \mathrm{eV}$ ). Introducing a correlation parameter $U$ on the Co site of around $3 \mathrm{eV}$ reduced $k_{3,1}$ to a value similar to the experimental one.

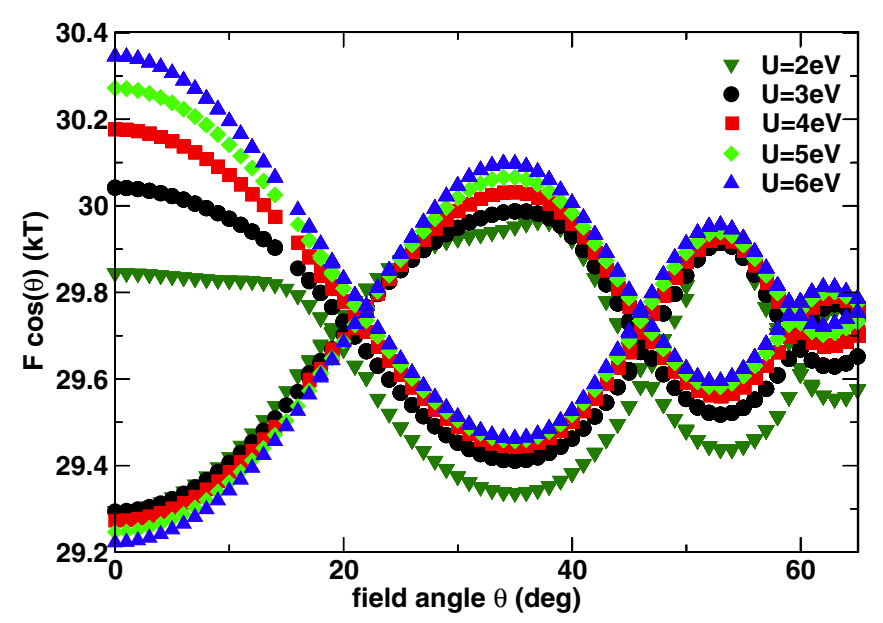

FIG. 7. Angular dependence of the DFT-calculated Fermi surface cross sections, multiplied by $\cos (\theta)$ for the $\mathrm{Z} \Gamma \mathrm{L}$ tilting plane. The frequencies depend significantly on the Coulomb parameter $U$ that is used to describe the strong correlations in the Co $3 d$ orbitals.

\section{E. Band-structure calculations for $\mathrm{PtCoO}_{2}$}

Density-functional calculations, applying GGA for the exchange correlation potential and including spin-orbit coupling, describe the Fermi surface of $\mathrm{PtCoO}_{2}$ rather well [22]. The result is a single band crossing the Fermi energy which is predominantly derived from Pt $5 d$ states. This band shows a weak dispersion along the $z$ direction, resulting in a Fermi surface shaped like a hexagonal cylinder (see Fig. 6). However, this band also shows a sizable admixture from Co $3 d$ states, leading to bumps at the edges of the hexagonal cylindershaped Fermi surface (see Fig. 6, upper left panel). These bumps in the calculations are characterized by their low Fermi velocities due to the small band width of the related Co states. In an ARPES experiment, these bumps were not observed [22], leading to the conclusion that they are artifacts and result from the underestimation of the strong correlations of the Co $3 d$ states in the insulating $\mathrm{CoO}_{2}$ layer by the DFT calculations. Applying DFT $+U$ as a mean-field description for the strong Coulomb repulsion in these orbitals, the experimental shape of the Fermi surface could be reproduced within the resolution of the ARPES experiment for a moderate value of $U=4 \mathrm{eV}$.

In contrast to the ARPES experiment, which provides a two-dimensional projection of the Fermi surface, the angulardependent $\mathrm{dHvA}$ measurements yield a highly precise scan of the three-dimensional Fermi surface. This enables a more detailed study of the role of correlations in the $\mathrm{CoO}_{2}$ layer and their influence on the Pt-derived Fermi surface. Figure 7 shows the calculated angular-dependent dHvA frequencies as a function of the value of $U$ for the Co $3 d$ states. We also investigated the influence of the Hund's exchange $J$ on the Fermi surface cross sections. However, the results for $J=0.6 \mathrm{eV}$ and $J=1.0 \mathrm{eV}$ were essentially identical.

Overall, the curves reproduce the experimental data very well (see Fig. 3). At first glance, they are rather similar in absolute frequency values, showing deviations from each other of the order of $1 \%$, only. This is expected since the averaged frequency is essentially fixed by the choice of lattice parameters due to the half-filled nature of the hexagonal 

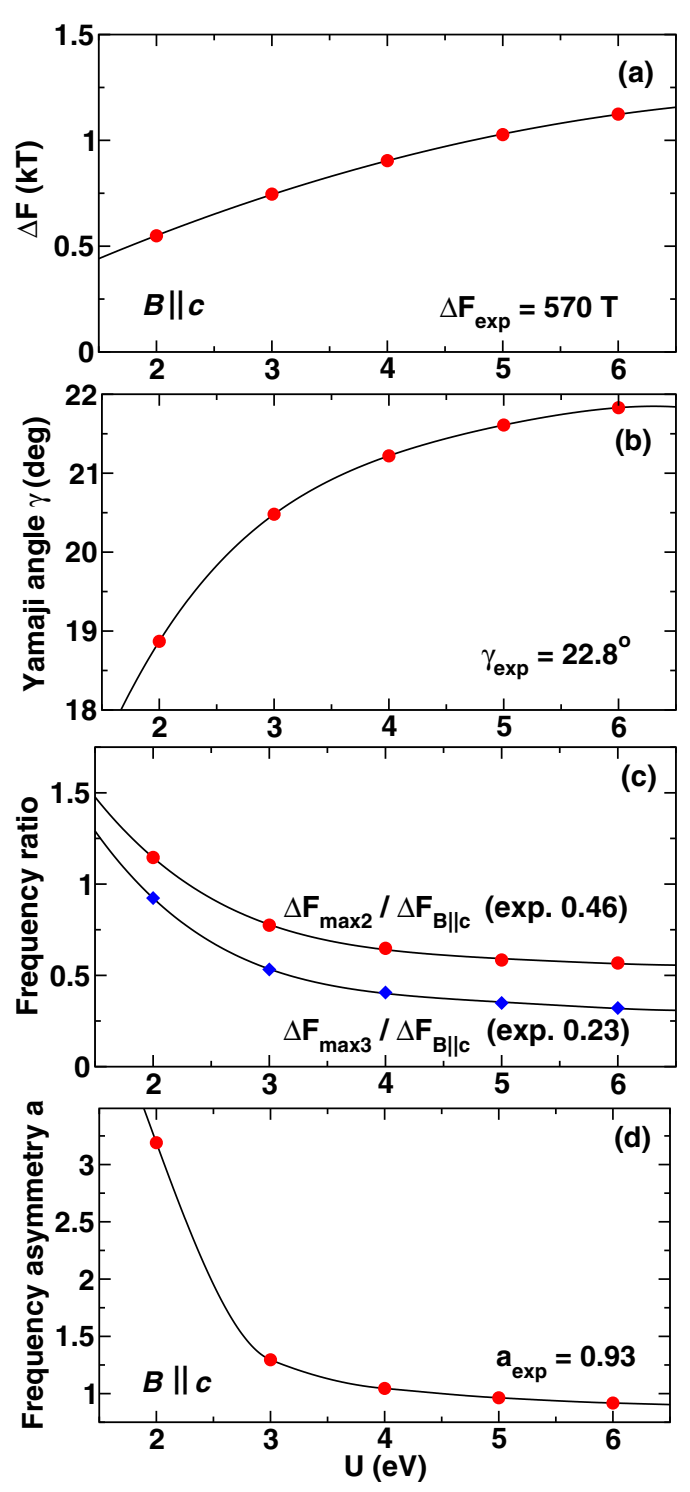

FIG. 8. Dependence of the calculated Fermi-surface properties on the Coulomb parameter $U$. Lines are guides to the eye. The respective experimental results are provided in the individual panels for comparison. Panel (a) shows the frequency split for the magnetic field $B \| c$. The Yamaji angle is shown in (b). Panel (c) provides the ratios for the second and third maximum frequency split with respect to the split for $B \| c$ in panel (a). Note that the second maximum split $\Delta F_{\max 2}$ is at around $35^{\circ}$ and the third maximum split $\Delta F_{\max 3}$ at around $53^{\circ}$ in the $\mathrm{Z} \Gamma \mathrm{L}$ tilting plane, compare Fig. 7. Panel (d) provides the asymmetry $a$ of the latter frequency split with respect to the frequency at the respective Yamaji angle. The definition of $a$ is given in the main text.

cylindrical Fermi surface. However, the plot shows a significant dependence of the frequency splits and the Yamaji angle on the $U$ parameter, which will be investigated now.

Comparing the shape of the calculated angular dependencies with the experiment (see Fig. 3), the agreement for small $U$ values (less than $3 \mathrm{eV}$ ) is rather poor. Especially, the flattening of the upper frequency at small angles and near the second maximum at around $35^{\circ}$ for $U=2 \mathrm{eV}$ is not reflected in the experiment. This is in line with the still

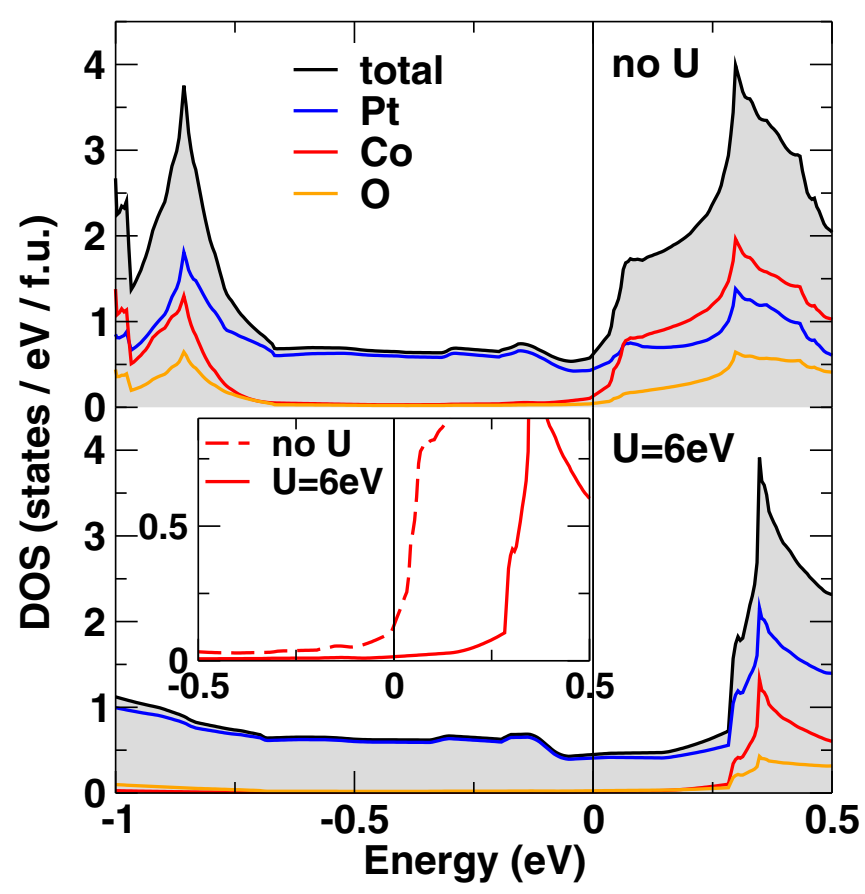

FIG. 9. Total and partial electronic density of states of $\mathrm{PtCoO}_{2}$ near the Fermi level $\varepsilon_{\mathrm{F}}$ (set to zero energy). The upper panel shows the GGA result, the lower panel includes the strong Coulomb repulsion for the Co $3 d$ orbitals in a mean-field approximation (GGA $+U$ ), leading to a strong reduction of the Co contribution to the $\mathrm{Pt}$ dominated band crossing $\varepsilon_{\mathrm{F}}$. The inset shows a direct comparison of the Co $3 d$ states for both scenarios.

significant Co $3 d$ contribution to the Fermi surface (see Fig. 6, upper right panel with $U=2 \mathrm{eV}$ ). The details of the Fermisurface warping depend on the choice of $U$ as presented in Fig. 8 showing (a) the frequency split for $B \| c$, (b) the Yamaji angle (the angle where the frequency split becomes zero), (c) the ratio of the split maxima at different angles, and (d) the split asymmetry $a$ for $B \| c\left(a=\left(F_{Y}-F_{\alpha}\right) /\left(F_{\beta}-F_{Y}\right)\right.$, where $F_{\alpha}$ and $F_{\beta}$ are the lower and the upper frequency for $B \| c$, respectively, and $F_{Y}$ is the frequency at the Yamaji angle) together with the respective experimental values. Essentially, all plots show a saturation for $U>6 \mathrm{eV}$. The latter three yield good agreement with the experiment for larger $U$ values. Similarly, the calculated masses show better agreement with the experiment for larger $U$ values. For $U=2 \mathrm{eV}$, due to the to overestimated hybridization with the Co $3 d$ states, we obtain $m_{\alpha}>m_{\beta}$ in contrast to the experiment. For $U=6 \mathrm{eV}$, we find $m_{\beta}-m_{\alpha}=0.09 m_{0}$ in excellent agreement with the measurements, although slightly smaller $U$ values are also in agreement with the experimental effective masses due to the large experimental uncertainties.

The origin of the significant $U$ dependence is an overestimate of the contribution of the Co $3 d$ states to the Pt-derived Fermi surface. Figure 9 shows the total and partial electronic density of states of $\mathrm{PtCoO}_{2}$ near the Fermi level $\varepsilon_{\mathrm{F}}$ for $U=$ $0 \mathrm{eV}$ and $U=6 \mathrm{eV}$. Although the absolute contribution of the Co $3 d$ states is only about $20 \%$ at $\varepsilon_{\mathrm{F}}$ for $U=0 \mathrm{eV}$ (see upper panel Fig. 9), they still have a significant influence on the shape of the Fermi surface due to their hybridization with the Pt band. This hybridization of the Co $3 d$ states at 
$\varepsilon_{\mathrm{F}}$ leads to the Fermi surface bumps with the low Fermi velocities due to the large admixture of Co $3 d$ orbitals for these parts of the $k$ space (compare Fig. 6). The application of $U(U=6 \mathrm{eV})$ increases the split between the occupied Co $t_{2 g}$ and the unoccupied $e_{g}$ states. Consequently, the upward shift of the $e_{g}$ band edge (see lower panel Fig. 9) leads to a strong reduction of the Co contribution by nearly a factor of 10 at $\varepsilon_{\mathrm{F}}$ (see insert Fig. 9), visible also in the disappearance of the "bumps" of the Fermi surface (see Fig. 6).

In contrast to the above-discussed improvements for a larger $U$ value, the frequency split for $B \| c$ is overestimated by a factor of about 2 for $U=6 \mathrm{eV}$. This overestimate can be directly linked to the dispersion due to the Pt interlayer coupling $t_{\perp}$. From our calculation, directly estimating the dispersion of the half-filled band at the Fermi level, we extract $t_{\perp}=55 \mathrm{meV}$ (for $U=6 \mathrm{eV}$ ) compared to $t_{\perp}=31 \mathrm{meV}$ from the experiment. The origin of this DFT overestimation is unclear, but similar effects have been observed for many other low-dimensional compounds for the couplings in the "weak" directions [36,37].

As result of our DFT calculations, we find good agreement between the calculated and the experimental Fermi surface when we include strong correlations for the Co $3 d$ states $(U=$ $6 \mathrm{eV}$ ). The assignment [22] of a larger $U$ value compared to the ARPES description with $U=4 \mathrm{eV}$ results from the greater sensitivity of the dHvA data with respect to the choice of $U$ and points to a strongly correlated regime in the $\mathrm{CoO}_{2}$ layers of $\mathrm{PtCoO}_{2}$.

Regardless of the details, our analysis demonstrates that strong correlations in the transition-metal layers play an important role in determining the properties of not just magnetic transition-metal ions $[20,21,38]$ but also of nonmagnetic ones like $\mathrm{Co}$ in $\mathrm{PtCoO}_{2}$.

\section{SUMMARY AND CONCLUSION}

This quantum-oscillation study combined with band-structure calculations gives detailed information on the Fermi surface of $\mathrm{PtCoO}_{2}$. Like the sister compounds
$\mathrm{PdCoO}_{2}$ and $\mathrm{PdRhO}_{2}$, it is a half-filled metal with a single quasi-two-dimensional Fermi surface with hexagonal cross-section, in the case of $\mathrm{PtCoO}_{2}$ formed by Pt states. The effective masses are near the free-electron value. By following the angle dependence of the two main quantum-oscillation frequencies, the warping parameters of the Fermi surface along the out-of-plane direction were determined. The warping along the $c$ axis is small, but its details enable an investigation of correlation effects in the $\mathrm{CoO}_{2}$ layers on the Fermi surface by comparing the experimental result with state-of-the art electronic-structure calculations. Many of the specific warping details such as the Yamaji angle and the ratio between first, second, and third frequency-split maximum with increasing angle, can be reproduced quantitatively by the calculations, when a strong Coulomb repulsion of $U=6 \mathrm{eV}$ is included in the $\mathrm{CoO}_{2}$ layers. The high precision and accuracy of dHvA data, combined with the effects of interlayer coupling, thus allow this study of the Fermi surface to yield information on the physics of strong correlations in the insulating $\mathrm{CoO}_{2}$ spacer layers.

\section{ACKNOWLEDGMENTS}

We thank Veronika Sunko, Clifford Hicks, Philip Moll, Pallavi Kushwaha, Phil King, Nigel Cooper, and Johannes Knolle for enriching discussions. The authors would like to acknowledge the financial support from the Max-Planck Society. E.H. and M.N. acknowledge support from Deutsche Forschungsgemeinschaft (DFG) through the Project No. 107745057 (TRR80: From Electronic Correlations to Functionality). We are grateful to late Professor James S. Brooks (NHMFL, Florida State University) for his continuous support and encouragement during this work. L.B. is supported by DOE-BES through Award No. DE-SC0002613. This work is also supported by JSPS KAKENHI (No. 18K04715). A portion of this work was performed at the National High Magnetic Field Laboratory, which is supported by the National Science Foundation Cooperative Agreements No. DMR1157490 and No. DMR-1644779 and the State of Florida.
[1] C. T. Prewitt, R. D. Shannon, and D. B. Rogers, Chemistry of noble metal oxides. 2. Crystal structure of $\mathrm{PtCoO}_{2}, \mathrm{PdCoO}_{2}$, $\mathrm{CuFeO}_{2}$ and $\mathrm{AgFeO}_{2}$, Inorg. Chem. 10, 719 (1971).

[2] R. D. Shannon, D. B. Rogers, and C. T. Prewitt, Chemistry of noble metal oxides. 1. Synthesis and properties of $\mathrm{ABO}_{2}$ delafossite compounds, Inorg. Chem. 10, 713 (1971).

[3] A. P. Mackenzie, The properties of ultrapure delafossite metals, Rep. Prog. Phys. 80, 032501 (2017).

[4] A. I. Coldea, L. Seabra, A. McCollam, A. Carrington, L. Malone, A. F. Bangura, D. Vignolles, P. G. van Rhee, R. D. McDonald, T. Soergel, M. Jansen, N. Shannon, and R. Coldea, Cascade of field-induced magnetic transitions in a frustrated antiferromagnetic metal, Phys. Rev. B 90, 020401 (2014).

[5] $\mathrm{AgNiO}_{2}$ is also metallic, but as the result of charge order in the $\mathrm{B}$ site Ni layer, is distinct from the monovalent metals discussed here. See A. I. Coldea, A. Carrington, R. Coldea, L. Malone,
A. F. Bangura, M. D. Johannes, I. I. Mazin, E. A. Yelland, J. G. Analytis, J. A. A. J. Perenboom, C. Jaudet, D. Vignolles, T. Sorgel, and M. Jansen, Interplay between localized and itinerant $d$ electrons in a frustrated metallic antiferromagnet, 2h- $\mathrm{AgNiO}_{2}$, arXiv:0908.4169.

[6] H. Takatsu, S. Yonezawa, S. Mouri, S. Nakatsuji, K. Tanaka, and Y. Maeno, Roles of high-frequency optical phonons in the physical properties of the conductive delafossite $\mathrm{PdCoO}_{2}$, J. Phys. Soc. Jpn. 76, 104701 (2007).

[7] H. Takatsu, S. Yonezawa, S. Fujimoto, and Y. Maeno, Unconventional Anomalous Hall Effect in the Metallic TriangularLattice Magnet $\mathrm{PdCrO}_{2}$, Phys. Rev. Lett. 105, 137201 (2010).

[8] C. W. Hicks, A. S. Gibbs, A. P. Mackenzie, H. Takatsu, Y. Maeno, and E. A. Yelland, Quantum Oscillations and High Carrier Mobility in the Delafossite $\mathrm{PdCoO}_{2}$, Phys. Rev. Lett. 109, 116401 (2012). 
[9] P. Kushwaha, V. Sunko, P. J. W. Moll, L. Bawden, J. M. Riley, N. Nandi, H. Rosner, M. P. Schmidt, F. Arnold, E. Hassinger, T. K. Kim, M. Hoesch, A. P. Mackenzie, and P. D. C. King, Nearly free electrons in a $5 d$ delafossite oxide metal, Sci. Adv. 1, e1500692 (2015).

[10] K. Kim, H. C. Choi, and B. I. Min, Fermi surface and surface electronic structure of delafossite $\mathrm{PdCoO}_{2}$, Phys. Rev. B 80, 035116 (2009).

[11] H. Takatsu, J. J. Ishikawa, S. Yonezawa, H. Yoshino, T. Shishidou, T. Oguchi, K. Murata, and Y. Maeno, Extremely Large Magnetoresistance in the Nonmagnetic Metal $\mathrm{PdCoO}_{2}$, Phys. Rev. Lett. 111, 056601 (2013).

[12] H.-J. Noh, J. Jeong, B. Chang, D. Jeong, H. S. Moon, E.-J. Cho, J. M. Ok, J. S. Kim, K. Kim, B. I. Min, H.-K. Lee, J.-Y. Kim, B.G. Park, H.-D. Kim, and S. Lee, Direct observation of localized spin antiferromagnetic transition in $\mathrm{PdCrO}_{2}$ by angle-resolved photoemission spectroscopy, Sci. Rep. 4, 3680 (2014).

[13] N. Kikugawa, P. Goswami, A. Kiswandhi, E. S. Choi, D. Graf, R. E. Baumbach, J. S. Brooks, K. Sugii, Y. Iida, M. Nishio, S. Uji, T. Terashima, P. M. C. Rourke, N. E. Hussey, H. Takatsu, S. Yonezawa, Y. Maeno, and L. Balicas, Interplanar coupling-dependent magnetoresistivity in high-purity layered metals, Nat. Commun. 7, 10903 (2016).

[14] P. J. W. Moll, P. Kushwaha, N. Nandi, B. Schmidt, and A. P. Mackenzie, Evidence for hydrodynamic electron flow in $\mathrm{PdCoO}_{2}$, Science 351, 1061 (2016).

[15] V. Sunko, H. Rosner, P. Kushwaha, S. Khim, F. Mazzola, L. Bawden, O. J. Clark, J. M. Riley, D. Kasinathan, M. W. Haverkort, T. K. Kim, M. Hoesch, J. Fujii, I. Vobornik, A. P. Mackenzie, and P. D. C. King, Maximal Rashba-like spin splitting via kinetic-energy- coupled inversion-symmetry breaking, Nature 549, 492 (2017).

[16] F. Mazzola, V. Sunko, S. Khim, H. Rosner, P. Kushwaha, O. J. Clark, L. Bawden, I. Markovic, T. K. Kim, M. Hoesch, A. P. Mackenzie, and P. D. C. King, Itinerant ferromagnetism of the Pd-terminated polar surface of $\mathrm{PdCoO}_{2}$, Proc. Nat. Acad. Sci. 115, 12956 (2018).

[17] H.-J. Noh, J. Jeong, J. Jeong, E.-J. Cho, S. B. Kim, K. Kim, B. I. Min, and H.-D. Kim, Anisotropic Electric Conductivity of Delafossite $\mathrm{PdCoO}_{2}$ Studied by Angle-Resolved Photoemission Spectroscopy, Phys. Rev. Lett. 102, 256404 (2009).

[18] J. A. Sobota, K. Kim, H. Takatsu, M. Hashimoto, S.-K. Mo, Z. Hussain, T. Oguchi, T. Shishidou, Y. Maeno, B. I. Min, and Z.-X. Shen, Electronic structure of the metallic antiferromagnet $\mathrm{PdCrO}_{2}$ measured by angle- resolved photoemission spectroscopy, Phys. Rev. B 88, 125109 (2013).

[19] F. Arnold, M. Naumann, S. Khim, H. Rosner, V. Sunko, F. Mazzola, P. D. C. King, A. P. Mackenzie, and E. Hassinger, Quasi-two-dimensional Fermi surface topography of the delafossite $\mathrm{PdRhO}_{2}$, Phys. Rev. B 96, 075163 (2017).

[20] J. M. Ok, Y. J. Jo, K. Kim, T. Shishidou, E. S. Choi, H.-J. Noh, T. Oguchi, B. I. Min, and J. S. Kim, Quantum Oscillations of the Metallic Triangular-Lattice Antiferromagnet $\mathrm{PdCrO}_{2}$, Phys. Rev. Lett. 111, 176405 (2013).

[21] C. W. Hicks, A. S. Gibbs, L. Zhao, P. Kushwaha, H. Borrmann, A. P. Mackenzie, H. Takatsu, S. Yonezawa, Y. Maeno, and E. A. Yelland, Quantum oscillations and magnetic reconstruction in the delafossite $\mathrm{PdCrO}_{2}$, Phys. Rev. B 92, 014425 (2015).
[22] P. Kushwaha, H. Borrmann, S. Khim, H. Rosner, P. J. W. Moll, D. A. Sokolov, V. Sunko, Yu. Grin, and A. P. Mackenzie, Single crystal growth, structure, and electronic properties of metallic delafossite $\mathrm{PdRhO}_{2}$, Cryst. Growth Des. 17, 4144 (2017).

[23] F. Arnold, M. Naumann, Th. Luehmann, A. P. Mackenzie, and E. Hassinger, Application of SQUIDs to low temperature and high magnetic field measurements-Ultra low noise torque magnetometry, Rev. Sci. Instrum. 89, 023901 (2018).

[24] C. Rossel, P. Bauer, D. Zech, J. Hofer, M. Willemin, and H. Keller, Active microlevers as miniature torque magnetometers, J. Appl. Phys. 79, 8166 (1996).

[25] K. Koepernik and H. Eschrig, Full-potential nonorthogonal local-orbital minimum-basis band-structure scheme, Phys. Rev. B 59, 1743 (1999).

[26] I. Opahle, K. Koepernik, and H. Eschrig, Full-potential bandstructure calculation of iron pyrite, Phys. Rev. B 60, 14035 (1999).

[27] J. P. Perdew, K. Burke, and M. Ernzerhof, Generalized Gradient Approximation Made Simple, Phys. Rev. Lett. 77, 3865 (1996).

[28] H. Eschrig, M. Richter, and I. Opahle, Relativistic solid state calculations, Relativistic Structure Theory, Part II: Applications, Theoretical and Computational Chemistry, Vol. 14 (Elsevier, Amsterdam, 2004).

[29] D. Shoenberg, Magnetic Oscillations in Metals (Cambridge University Press, Cambridge, 2009).

[30] N. W. Ashcroft and D. M. Mermin, Festkoerperphysik, 3rd ed. (Oldenbourg Verlag, München, 2007).

[31] M. V. Kartsovnik, P. D. Grigoriev, W. Biberacher, N. D. Kushch, and P. Wyder, Slow Oscillations of Magnetoresistance in Quasi-Two-Dimensional Metals, Phys. Rev. Lett. 89, 126802 (2002).

[32] P. D. Grigoriev, Theory of the Shubnikov-de Haas effect in quasi-two-dimensional metals, Phys. Rev. B 67, 144401 (2003).

[33] P. D. Grigoriev, The influence of the chemical potential oscillations on the de Haas-van Alphen effect in quasi-two- dimensional compounds, JETP 92, 1090 (2001).

[34] C. Bergemann, S. R. Julian, A. P. Mackenzie, S. NishiZaki, and Y. Maeno, Detailed Topography of the Fermi Surface of $\mathrm{Sr}_{2} \mathrm{RuO}_{4}$, Phys. Rev. Lett. 84, 2662 (2000).

[35] C. Bergemann, A. P. Mackenzie, S. R. Julian, D. Foresythe, and E. Ohmichi, Quasi-two-dimensional Fermi liquid properties of the unconventional superconductor $\mathrm{Sr}_{2} \mathrm{RuO}_{4}$, Adv. Phys. 52, 639 (2003).

[36] H. Rosner, H. Eschrig, R. Hayn, S. L. Drechsler, and J. Malek, Electronic structure and magnetic properties of the linear chain cuprates $\mathrm{Sr}_{2} \mathrm{CuO}_{3}$ and $\mathrm{Ca}_{2} \mathrm{CuO}_{3}$, Phys. Rev. B 56, 3402 (1997).

[37] D. Kasinathan, K. Koepernik, and H. Rosner, Quasi-OneDimensional Magnetism Driven by Unusual Orbital Ordering in $\mathrm{CuSb}_{2} \mathrm{O}_{6}$, Phys. Rev. Lett. 100, 237202 (2008).

[38] V. Sunko, F. Mazzola, S. Kitamura, S. Khim, P. Kushwaha, O. J. Clark, M. Watson, I. Markovic, D. Biswas, L. Pourovskii, T. K. Kim, T. L. Lee, P. K. Thakur, H. Rosner, A. Georges, R. Moessner, T. Oka, A. P. Mackenzie, and P. D. C. King, Probing spin correlations using angle resolved photoemission in a coupled metallic/Mott insulator system, Sci. Adv. 6, eaaz0611 (2020). 